\title{
Molecular Modifiers Suppress Nonclassical Pathways of Zeolite Crystallization
}

\author{
Wei Qin, Ankur Agarwal, Madhuresh K. Choudhary, Jeremy C. Palmer, and Jeffrey D. Rimer* \\ Department of Chemical and Biomolecular Engineering, University of Houston, 4726 Calhoun \\ Road, Houston, TX 77204 USA \\ *Correspondence sent to: jrimer@central.uh.edu
}

\section{$\underline{\text { List of Figures }}$}

Figure S1. Mass spectra of an alkaline D-Arg solution before and after heating.

Figure S2. ${ }^{13} \mathrm{C}$ NMR spectrum of an alkaline D-Arg solution before and after heating.

Figure S3. ${ }^{13} \mathrm{C}$ NMR spectrum of ornithine-lactam (Racemic-OL).

Figure S4. ${ }^{13} \mathrm{C}$ NMR spectrum of an alkaline urea solution before and after heating.

Figure S5. ${ }^{13} \mathrm{C}$ NMR spectrum of an alkaline D-ornithine solution before and after heating.

Figure S6. Measurements of $\mathrm{pH}$ changes in solution $\mathrm{S}(\mathrm{I})$ as a function of R-OL concentration.

Figure S7. Silicalite-1 surfaces used in USMD simulations to investigate R/S-OL adsorption.

Figure S8. Powder XRD patterns of extracted solids from syntheses in S(II) growth solution.

Figure S9. Effect of R-OL on the morphology of ZSM-5 crystals. 


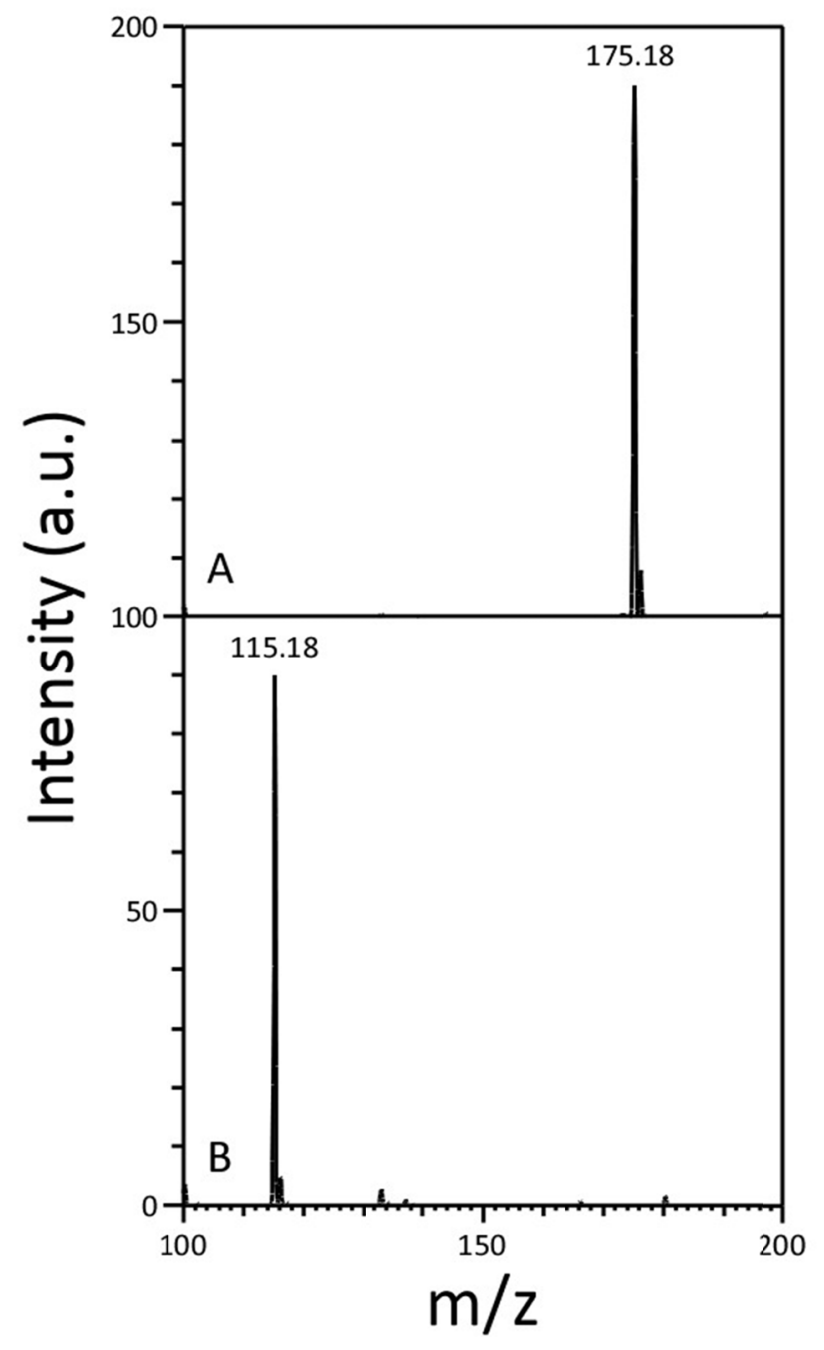

Figure S1. Mass spectra of a solution containing D-arginine and $\mathrm{NaOH}(\mathrm{pH}$ 12.3) that was measured (A) before and (B) after hydrothermal treatment at $160^{\circ} \mathrm{C}$ for $72 \mathrm{~h}$. The $\mathrm{m} / \mathrm{z}$ ratios correspond to D-arginine and (R)-ornithine-lactam, respectively. 


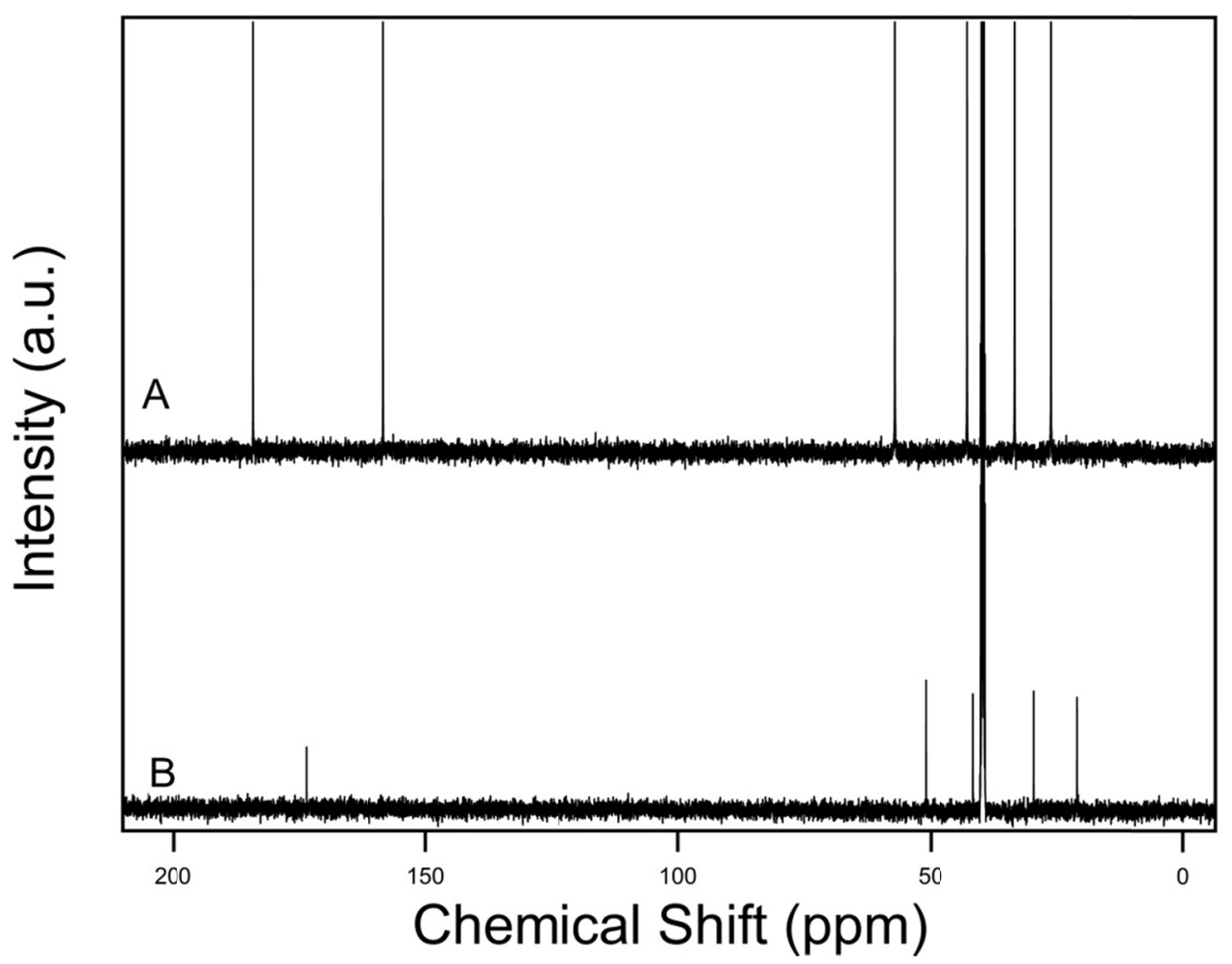

Figure S2. ${ }^{13} \mathrm{C}$-NMR spectrum in DMSO of (A) D-Arg and (B) organics that were extracted from a solution containing D-arginine and $\mathrm{NaOH}(\mathrm{pH} 12.3)$ after heating at $160^{\circ} \mathrm{C}$ for $72 \mathrm{~h}$. The pattern matches that of ornithine-lactam (see Figure S3). 


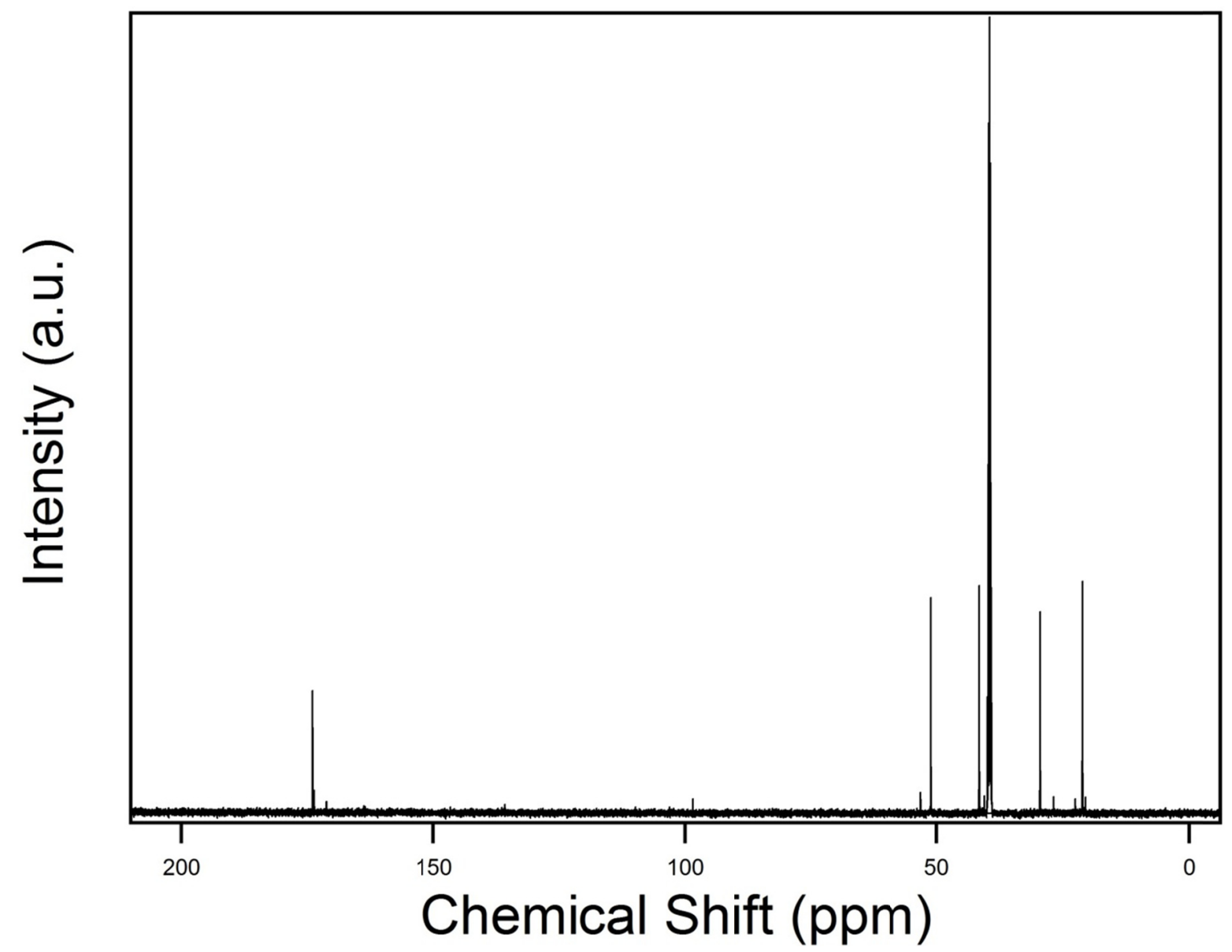

Figure S3. ${ }^{13} \mathrm{C}-\mathrm{NMR}$ spectrum of ornithine-lactam (R/S-OL) in DMSO. The reagent was purchased from AK Scientific Inc. to confirm the product of D-Arg. thermal decomposition. 


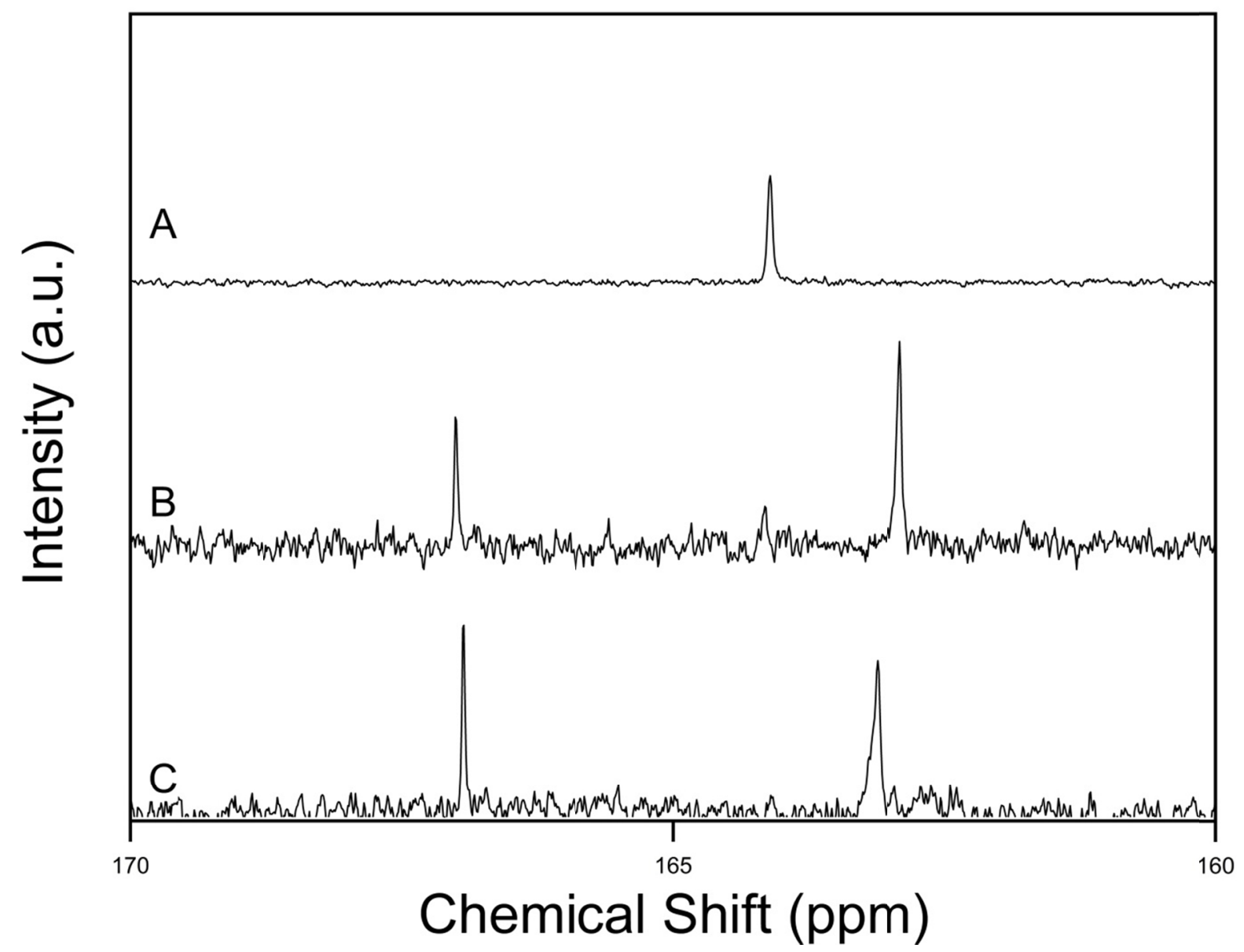

Figure S4. ${ }^{13} \mathrm{C}$-NMR spectrum of urea measured in alkaline solution (A) without heating (i.e. as received reagent from EMD Chemicals Inc.), and after hydrothermal treatment at $160^{\circ} \mathrm{C}$ for (B) $4 \mathrm{~h}$ and (C) $8 \mathrm{~h}$. The final product of urea decomposition is carbonate. 


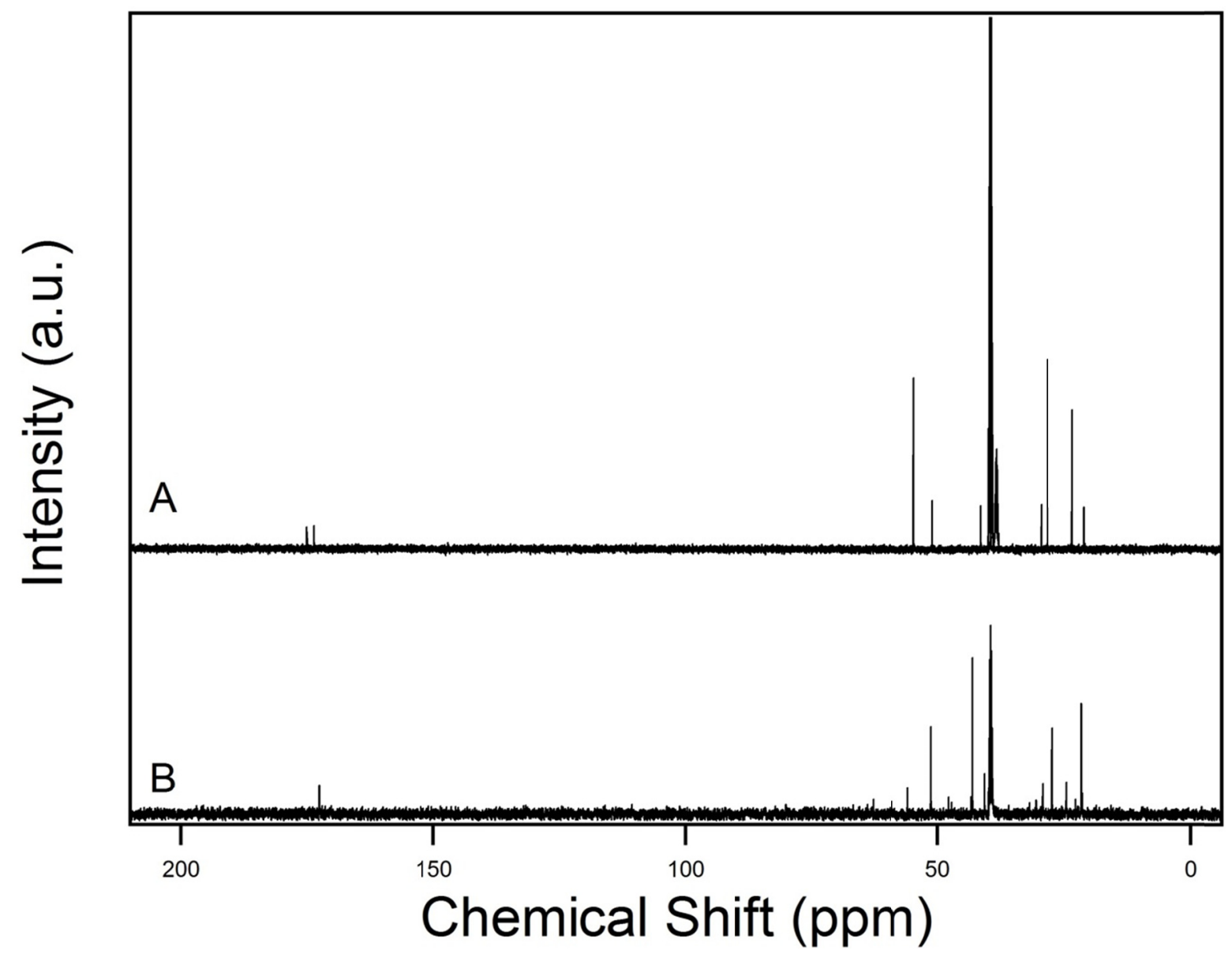

Figure S5. ${ }^{13} \mathrm{C}$-NMR spectrum of D-ornithine measured in alkaline solution (A) without heating (i.e. as received reagent from Sigma Aldrich), and (B) after hydrothermal treatment at $160^{\circ} \mathrm{C}$ for $8 \mathrm{~h}$. The pattern matches that of ornithine-lactam (see Figure S3). 


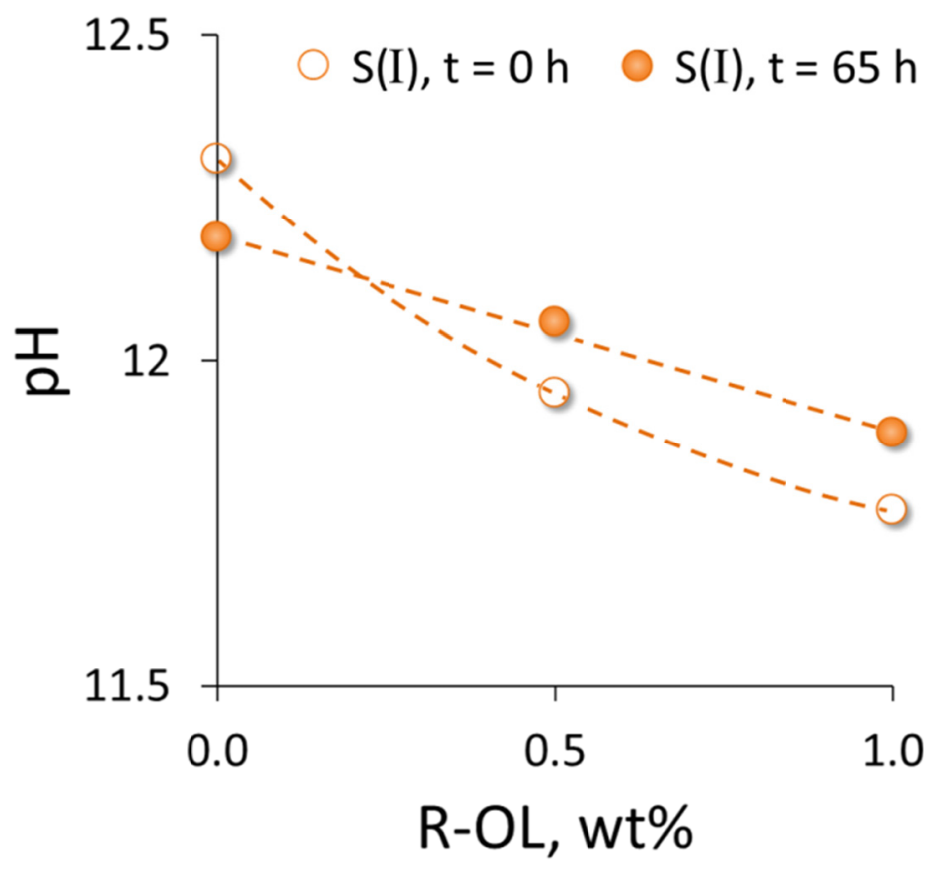

Figure S6. Measurements of $\mathrm{pH}$ for silicalite-1 growth solution $\mathrm{S}(\mathrm{I})$ prior to $\left(\mathrm{pH}_{\mathrm{i}}\right.$, open symbols) and after $\left(\mathrm{pH}_{\mathrm{o}}\right.$, solid symbols) hydrothermal treatment at $160^{\circ} \mathrm{C}$ for $65 \mathrm{~h}$ as a function of R-OL weight percentage. The dashed lines are interpolated to help guide the eye. 

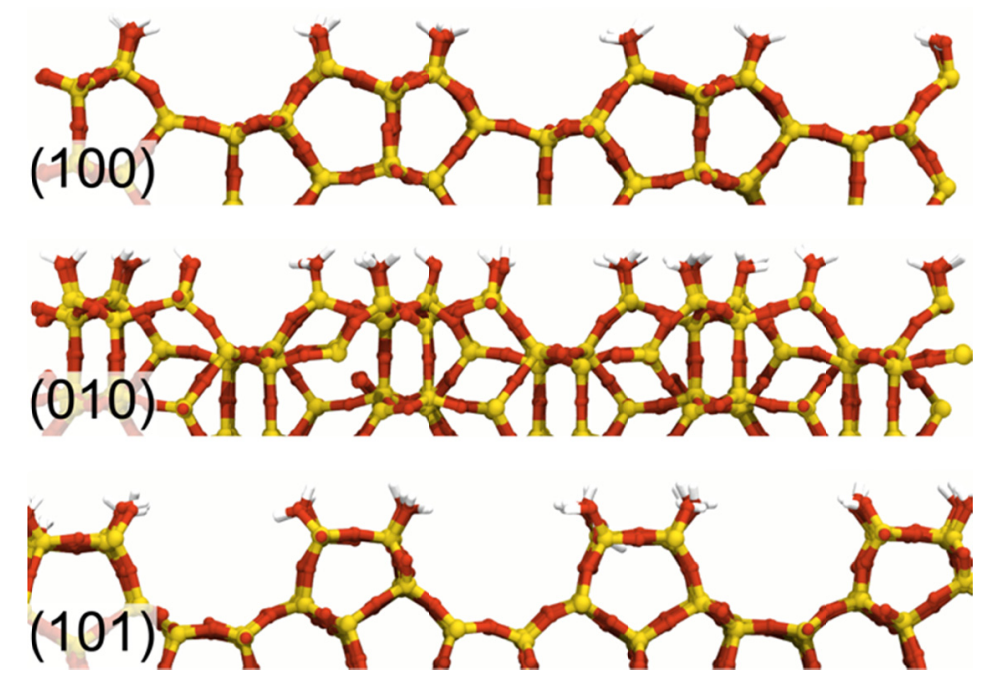

Figure S7. Model (hkl) surfaces of silicalite-1 used in the USMD simulations to investigate R/SOL adsorption. 


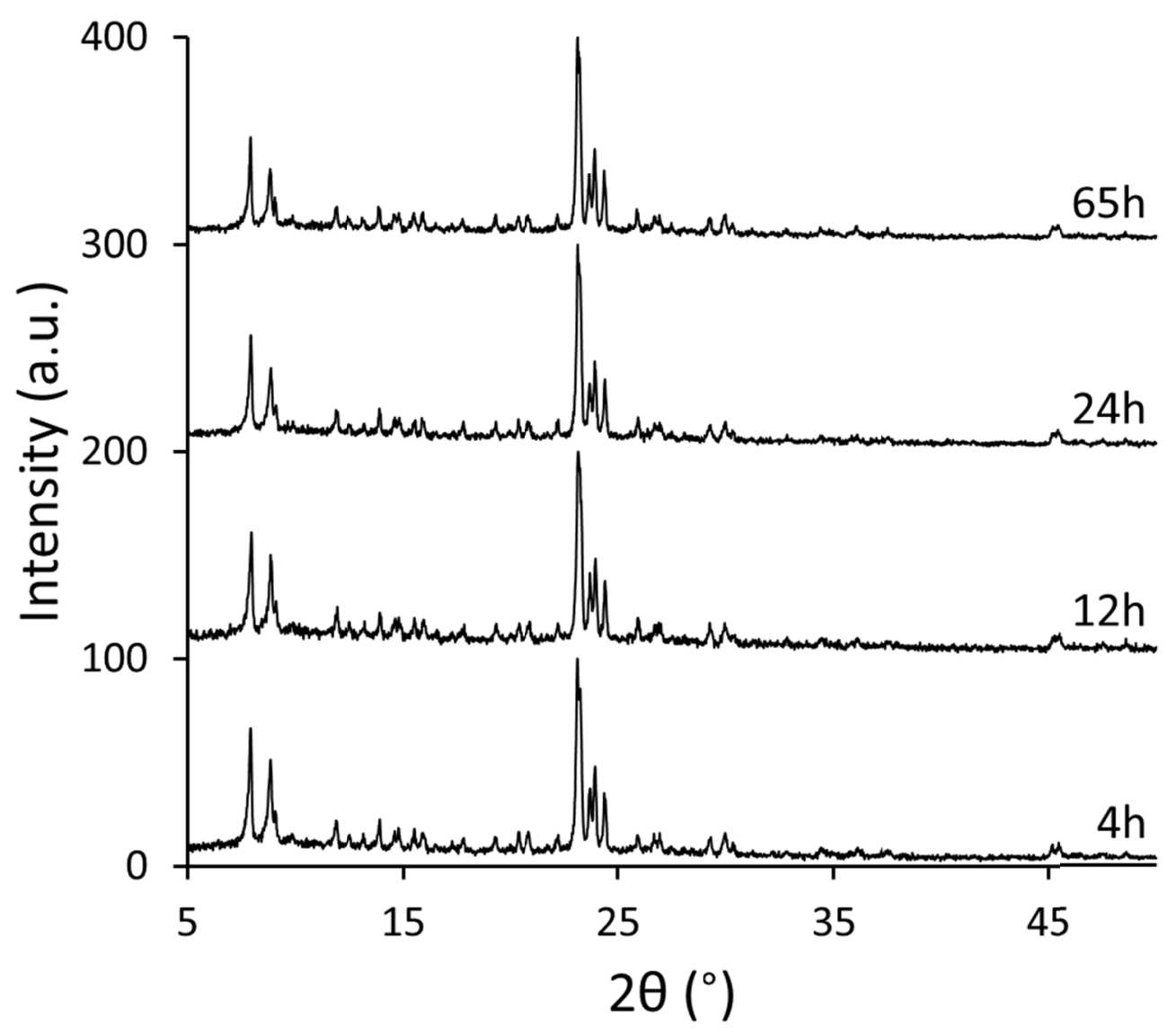

Figure S8. Powder XRD patterns of extracted solids from S(II) growth solutions after 4, 12, 24, and $65 \mathrm{~h}$ of hydrothermal treatment at $160^{\circ} \mathrm{C}$. 
Growth modification of ZSM-5. The effect of R-OL on ZSM-5 (MFI type) crystal morphology and size was assessed by bulk crystallization studies in both the absence of modifier and in the presence of varying R-OL weight fraction. We added aluminum sulfate $\left(\mathrm{Al}_{2}\left(\mathrm{SO}_{4}\right)_{3}\right)$ to solution $\mathrm{S}(\mathrm{II})^{\prime}$ to make an initial gel $\mathrm{Si} / \mathrm{Al}$ ratio of 50 . The majority of aluminosilicate species are in the form of nanoparticle precursors. ZSM-5 crystals produced from growth solution S(II)' (containing Al) in the absence of modifier exhibit a typical ZSM-5 morphology (Figure S9A). A systematic study of varying modifier content (Figure S9C) reveals little change in crystal thickness, while crystal length increases monotonically with increasing D-Arg concentration. The aspect ratio of length over thickness increases with increasing modifier concentration (Figure S9B). This agrees with the observations for silicalite-1 crystals synthesized in solution S(II).

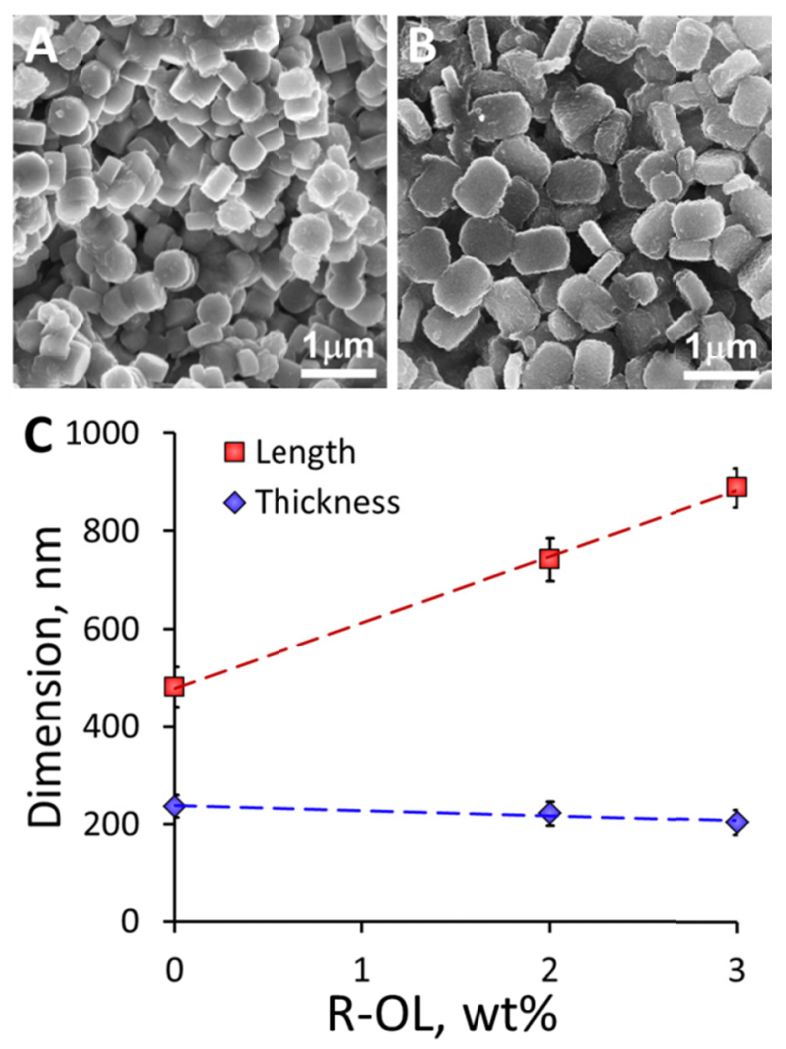

Figure S9. Effect of R-OL (i.e. the decomposition product of D-Arg) on the morphology of ZSM-5 crystals synthesized from growth solutions with molar composition $157 \mathrm{SiO}_{2}: 1.57$ $\mathrm{Al}_{2} \mathrm{O}_{3}: 47$ TPAOH. Scanning electron micrographs of ZSM-5 crystals prepared with the following synthesis conditions: (A) control (absence of D-Arg) and (B) $3 \mathrm{wt} \% \mathrm{D}-\mathrm{Arg}$. (C) Plot of crystal length (red squares) and thickness (blue triangles) of ZSM-5 crystals as a function of modifier concentration. Crystal dimensions are obtained from SEM images and each value is the 
average of more than 50 measurements from a single crystal batch. Dashed lines are interpolated to help guide the eye, and error bars equal two standard deviations. 